\title{
Osteopontin depletion decreases inflammation and gastric epithelial proliferation during Helicobacter pylori infection in mice
}

\author{
Jun Won Park ${ }^{1,2,4}$, Su Hyung Lee ${ }^{1,4}$, Du Min Go ${ }^{1}$, Hark Kyun Kim², Hyo-Jung Kwon ${ }^{3}$ and Dae-Yong Kim
}

Osteopontin (OPN) is a multifunctional protein that plays a role in many physiological and pathological processes, including inflammation and tumorigenesis. Here, we investigated the involvement of OPN in Helicobacter pylori (HP)-induced gastritis using OPN knockout (KO) mice and OPN knockdown (KD) cell lines. HP-infected OPN KO mice showed significantly reduced gastritis compared with wild-type (WT) mice with decreased infiltration of macrophages and a reduction in HP-induced upregulation of IL-1 $\beta$, TNF- $\alpha$, and IFN- $\gamma$. HP-exposed OPN KD gastric cancer cells and macrophage-like cells showed an attenuated induction of these cytokines. We also demonstrated a reduction in the migration of monocytic and macrophage-like cells toward conditioned media harvested from HP-exposed OPN KD gastric cancer cells as well as reduced migration ability of OPN KD cells itself. In addition, HP-infected OPN KO mice showed decreased epithelial cell proliferation compared with HP-infected WT mice, in association with a reduction in MAPK pathway activation. OPN KD gastric cancer cell lines also showed lower proliferative activity and reduced MAPK activation than shRNA control cells after HP co-culture or after IL-1 $\beta$ and TNF- $\alpha$ treatment. Taken together, these results indicate that OPN exerts a considerable influence on $H P$-induced gastritis by modulating the production of cytokines and contributing to macrophage infiltration. Moreover, OPN-mediated activation of the MAPK pathway in gastric epithelial cells might contribute to epithelial changes following HP infection.

Laboratory Investigation (2015) 95, 660-671; doi:10.1038/labinvest.2015.47; published online 13 April 2015

Helicobacter pylori $(H P)$ is a Gram-negative bacterial pathogen that selectively colonizes the human stomach. ${ }^{1,2}$ Approximately half of the world's population is infected with $H P$, and its infection is strongly associated with chronic gastritis, peptic ulcers, and gastric cancer. ${ }^{1,2} H P$-infected gastric mucosa progresses through stages of chronic gastritis, glandular atrophy, intestinal metaplasia, dysplasia, and gastric cancer. ${ }^{3}$ $H P$ produce a variety of virulence factors such as CagA and VacA that can affect host intracellular signaling pathways and play an important role in determining the outcome of $H P$ infection. ${ }^{3}$ However, they are not absolute determinants of clinical outcomes because most individuals remain asymptomatic after HP infection. ${ }^{4}$ The variable outcomes of $H P$ infection can be attributed to inflammatory responses governed by host factors as well as HP virulence factors. ${ }^{5}$ Indeed, polymorphisms of host interleukin-1 $\beta$ (IL-1 $\beta$ ) and tumor necrosis factor- $\alpha$ (TNF- $\alpha$ ) are reported to be associated with the risk of $H P$-associated gastric cancer development. ${ }^{4}$

$H P$-induced chronic inflammation induces DNA alterations and imbalanced epithelial cell turnover, providing opportunities for mitotic error that could ultimately contribute to the development of gastric cancer. ${ }^{3,6-8}$ Infiltrating macrophages, in particular, produce various cytokines and growth factors such as IL- $1 \beta$ that could attract more inflammatory cells and induces the proliferation of gastric epithelial cells. ${ }^{9,10}$ A recent study demonstrated that IL-1 $\beta$ physiologically induced by $H P$ infection enhanced gastric inflammation and the number of gastric tumors using $1 l 1 b$-mull mice. ${ }^{11}$ Moreover, host factors secreted from gastric epithelial cells, such as Sonic Hedgehog, could also be involved in the initiation of gastritis, acting as a macrophage chemoattractant during HP infection. ${ }^{12}$ The interaction between gastric epithelial cells

Department of Veterinary Pathology, College of Veterinary Medicine, Seoul National University, Seoul, Korea; ${ }^{2}$ National Cancer Center, Goyang, Gyeonggi, Korea and

${ }^{3}$ College of Veterinary Medicine, Chungnam National University, Daejeon, Korea

Correspondence: Professor D-Y Kim, DVM, PhD, Department of Veterinary Pathology, College of Veterinary Medicine, Seoul National University, 599 Gwanak-ro, Gwanak-gu, Seoul 151-742, Korea.

E-mail: daeyong@snu.ac.kr

${ }^{4}$ These authors contributed equally to this work.

Received 18 August 2014; revised 22 January 2015; accepted 28 January 2015 
and infiltrating macrophages during $H P$ infection might play an important role in the development of gastritis and $H P$-related pathogenic changes in gastric epithelial cells. Therefore, host factors involved in such interactions during $H P$ infection need to be further investigated.

Osteopontin (OPN), encoded by a secreted phosphoprotein 1 (SPP1) gene, is a multifunctional glycol-phosphoprotein that plays important roles in a wide range of pathophysiological processes, such as inflammation, angiogenesis, bone remodeling, and cell migration. ${ }^{6,7} \mathrm{OPN}$, functioning as chemoattractive Th1 cytokine, promotes the migration of inflammatory cells. ${ }^{7,13}$ Clinically, increased plasma OPN levels were proven in many chronic inflammatory diseases such as Crohn's disease and rheumatoid arthritis and various cancers including gastric cancer. ${ }^{7}$ A recent study showed that increased gastric OPN expression in response to HP infection correlates well with the degree of gastric inflammation and intestinal metaplasia in humans. ${ }^{14}$ However, the exact functional roles of OPN in HP-induced gastritis have not yet been fully investigated. Previous reports have focused on the role of OPN in macrophage functions in various immunological disorders, ${ }^{7}$ whereas the role of OPN in epithelial cells in response to infectious pathogens, such as $H P$, has been not studied.

In this study, we investigated the possible roles of OPN during $H P$-induced gastritis using OPN knockout $(\mathrm{KO})$ mice and stable OPN knockdown (KD) cell lines. The results showed that OPN is an important mediator of HP-induced gastritis and regulates cytokine production, macrophage migration, and proliferation of gastric epithelial cells in response to $H P$ infection. Our results provide important insights into the role of OPN in inflammatory processes and gastric epithelial changes that occur in response to HP infection.

\section{MATERIALS AND METHODS \\ Mice}

Female C57BL/6-Spp $1^{\mathrm{tm} 1 \mathrm{Blh}(-/-)}$ (OPN KO) mice were purchased from Jackson Laboratory (Bar Harbor, ME, USA) and wild-type (WT) littermates were purchased from Orientbio (Seongnam, Korea) at 5 weeks of age. Mouse studies were conducted with the approval of the Animal Care and Use Committees of Seoul National University (certification number: SNU-100729-1). The mice were used in the study after 1 week of adjustment to the facility.

\section{Cell Culture and Isolation of Peritoneal Macrophages}

Human gastric cancer cell line AGS, human monocytic cell line THP-1, and mouse macrophage-like cell line RAW264.7 were purchased from American Type Culture Collection (Manassas, VA, USA). NCC-S1M, mouse gastric cancer cell line, was primarily cultured from a spontaneous gastric carcinoma arising in a Villin-cre; $\operatorname{Trp} 53^{F / F} ; \operatorname{Smad} 4^{F / F} ; \mathrm{Cdh} 1^{F / w t}$ mouse. ${ }^{15}$ All the cell lines were cultured at $37^{\circ} \mathrm{C}$ in a $5 \% \mathrm{CO}_{2}$ humidified incubator in RPMI-1640 medium (Gibco, Grand Island, NY, USA) containing 10\% fetal bovine serum (Gibco),
$1 \%$ penicillin and streptomycin (Invitrogen Biotechnology, Grand Island, NY, USA), and $0.05 \mathrm{mM}$ mercaptoethanol was additionally supplemented to culture THP-1 cell line. To produce differentiated macrophage-like cells (Di-THP-1), THP-1 cells were seeded on the 6 -well plates at $1 \times 10^{6}$ cells with $200 \mathrm{nM}$ of phorbol 12-myristate 13-acetate (PMA; SigmaAldrich, St Louis, MO, USA) and incubated for $24 \mathrm{~h}$.

To isolate peritoneal macrophages from mice, we used previously described method. ${ }^{16}$ Briefly, female 5-week-old WT and OPN KO mice were injected intraperitoneally with $10 \mathrm{ml}$ cold PBS after killing by $\mathrm{CO}_{2}$ and the peritoneal fluid was withdrawn by syringe suction. Peritoneal cells in the fluid were allowed to adhere in tissue culture plates in cell culture media for $1 \mathrm{~h}$. Nonadherent cells were removed by washing with warm PBS. We verified $>85 \%$ macrophages in the preparation by flow cytometry using anti-mouse F4/80 antigen PE (1:500; 12-4801-80, eBioscience, San Diego, CA, USA).

\section{HP Infection and In Vitro Co-Culture}

Mouse-adapted HP Sydney strain 1 (SS1) was grown on brain heart infusion agar plates (Difco, Detroit, MI, USA) containing $10 \%$ sheep blood under microaerobic conditions produced by GasPak jars (Difco) and Campy-Paks (Becton Dickinson, Cockeysville, MD, USA) at $37^{\circ} \mathrm{C}$. After $24 \mathrm{~h}$ of fasting, 6-week-old OPN KO and WT mice $(n=20$ and 20, respectively) were orally administered with a $0.1-\mathrm{ml}$ suspension of $H P$ containing $1 \times 10^{9}$ colony-forming units $(\mathrm{CFUs}) / \mathrm{ml}$ for three times every other day in 1 week. Twenty additional mice in each group were used as sham-infected controls. Ten mice in each group were killed at 8 and 16 weeks after $H P$ infection, respectively. To quantify HP in mouse stomach, total DNA was extracted from mouse stomachs using QIAamp DNA mini kit (Qiagen, Hilden, Germany) according to the manufacturer's instructions and was amplified using specific primers for the 16S rDNA gene of HP SS1 with TaqMan probe. ${ }^{17}$ The quantity of HP $16 \mathrm{~S}$ gene was normalized to that of mouse GAPDH gene.

For the in vitro co-culture experiment, HP strain 60190 was used because of the low viability of HP SS1 in RPMI-1640 media and the similar pathogenicity with HP SS1 (CagA (+), VacA (+)). HP strain 60190 was kindly provided by Dr Yong Chan Lee (Department of Internal Medicine, Yonsei University, Korea). HP 60190 was grown according to the same method used for the cultivation of HP SS1. The conditions for HP co-culture have been described previously. ${ }^{14,18}$ Briefly, AGS, NCC-S1M, Di-THP-1, RAW264.7 cells, and peritoneal macrophages were seeded on 6-well plates at a density of $1.5 \times 10^{5}$ cells per well in RPMI-1640 medium with $10 \%$ FBS. Then, $12 \mathrm{~h}$ after seeding, the culture medium was replaced with RPMI-1640 containing 1\% FBS and no antibiotics. After $12 \mathrm{~h}, H P$ was suspended in the RPMI-1640 medium and added to the cells at a concentration of 10 multiplicity of infection (MOI). To verify the $H P$ viability in the co-culture condition, supernatants and scrapped gastric cancer cells after co-culture for $24 \mathrm{~h}$ were collected and grown on brain heart 
infusion agar plates containing $10 \%$ sheep blood under microaerobic conditions. After 3 days of incubation, $H P$ colonies were identified by colony morphology, urease activity, and PCR (data not shown). To prepare the conditioned media $(\mathrm{CM})$, the culture media were harvested at $24 \mathrm{~h}$ after $H P$ co-culture and filtered using a $0.45 \mu \mathrm{m}$ filter.

The cell proliferation was determined using the MTT assay. At $24 \mathrm{~h}$ after HP co-culture, $200 \mu \mathrm{l}$ of MTT solution $(5 \mathrm{mg} / \mathrm{ml}$ in PBS) was added to each well in $1 \mathrm{ml}$ of media. Formazane formation was terminated after $2 \mathrm{~h}$ by removing the MTT solution. Then, $500 \mu \mathrm{l}$ DMSO was added into each well to dissolve the precipitation. The absorbance was detected at $570 \mathrm{~nm}$ with a Microplate Reader.

Flow cytometry for cell cycle analysis was performed by using the co-cultured cells with HP for $24 \mathrm{~h}$. Cells were harvested, washed with PBS, fixed in $70 \%$ ethanol at $4{ }^{\circ} \mathrm{C}$ overnight, washed with cold PBS, and stained with propidium iodide (PI) staining solution $(50 \mu \mathrm{g} / \mathrm{ml}$ PI and $0.1 \mathrm{mg} / \mathrm{ml}$ RNase A in PBS) at RT for $30 \mathrm{~min}$. Cell cycles were then analyzed using FACSCalibur (BD Biosciences, San Jose, CA, USA).

\section{Necropsy and Histopathological Examination}

Mice were killed $24 \mathrm{~h}$ after fasting and stomachs were incised along the greater curvature. The stomach was spread onto a filter paper and cut in half along the lesser curvature. One part was stored in $\mathrm{a}-70^{\circ} \mathrm{C}$ deep freezer for subsequent analysis. The other part was fixed in neutral buffered $10 \%$ formalin for 1 day. Then, the formalin-fixed stomachs were cut into three strips and processed by standard method. The $5 \mu \mathrm{m}$ paraffin-embedded sections were stained with hematoxylin and eosin (H\&E). The degrees of mucosal inflammation after $H P$ infection were graded on the basis of previously delineated criteria. ${ }^{19}$

\section{Immunohistochemistry (IHC) and Double Immunofluorescence}

The replicate paraffin sections were dewaxed, rehydrated, and subjected to antigen retrieval by heating at $100{ }^{\circ} \mathrm{C}$ for $20 \mathrm{~min}$ in $0.01 \mathrm{M}$ citrate buffer ( $\mathrm{pH}$ 6.0). The ImmPRESS Peroxidase Polymer kit (Vector Laboratories, Burlingame, CA, USA) was used for immunostaining according to the manufacturer's protocol. Briefly, the slides were incubated with the $2.5 \%$ horse serum for blocking and then incubated for $30 \mathrm{~min}$ at room temperature with the primary antibodies. Rabbit polyclonal anti-Ki-67 (1:200; ab15580, Abcam, Cambridge, MA, USA), rabbit monoclonal anti-CD3 (1:100; ab16669, Abcam), rat monoclonal anti-F4/80 (1:200; ab6640, Abcam), mouse monoclonal anti-spasmolytic polypeptide antibody (TFF2) (1:50; ab49536, Abcam), and goat polyclonal antiOPN (1:100; AF808, R\&D systems, Minneapolis, MN, USA) were used as primary antibodies. After washing, the slides were then incubated for $30 \mathrm{~min}$ with peroxidase polymer-linked appropriate secondary antibodies. The slides were subjected to colorimetric detection with ImmPact DAB substrate
(SK-4105, Vector Laboratories). The slides were counterstained with Meyer's hematoxylin for $10 \mathrm{~s}$. Negative controls were performed by omitting the primary antibody and substitution with diluent.

For double immunofluorescence analysis, the slides were incubated with $5 \%$ goat serum for first blocking. The first primary antibody against F4/80 (1:100; ab6640, Abcam) was incubated overnight at $4{ }^{\circ} \mathrm{C}$. After washing, the Texas red goat anti-rat secondary antibody (1:250; TI-9400, Vector Laboratories) was incubated for $1 \mathrm{~h}$ at room temperature. Then, the slides were incubated with 5\% rabbit serum for second blocking. After blocking, second primary antibody against OPN (1:50; AF808, R\&D Systems) was incubated overnight for $1 \mathrm{~h}$ and the FITC rabbit anti-goat secondary antibody (1:250; FI-5000, Vector Laboratories) was incubated for $1 \mathrm{~h}$ at room temperature. Slides were mounted with Vectashield mounting media (H-1200, Vector Laboratories). Stained slides were evaluated using Zeiss Axio Imager HBO 100 (Carl Zeiss, Oberkochen, Germany).

\section{Lentiviral shRNA for Stable OPN Knockdown}

The lentiviral human SPP1 and mouse Spp1 shRNA constructs were purchased from Sigma-Aldrich with pLKO.1-puro eGFP control vector (SHC005, Sigma). The target constructs were generated from accession number NM_000582 (5'-CCGG CCGAGGTGATAGTGTGGTTTACTCGAGTAAACCACACT ATCACCTCGGTTTTT-3') and NM_009263 (5'-CCGGAGG ATGACTTTAAGCAAGAAACTCGAGTTTCTTGCTTAAAGT CATCCTTTTTTG-3'). Lentiviruses were produced by cotransfecting shRNA-expressing vector and pMD2.G and psPAX2 constructs (Addgene, Cambridge, MA, USA) into 293T cells by using Lipofectamine 2000 (Invitrogen). Viral supernatants were harvested $48 \mathrm{~h}$ after transfection, filtered though a $0.45 \mu \mathrm{m}$ filter, tittered, and used for viral transduction with $10 \mu \mathrm{g} / \mathrm{ml}$ polybrene. AGS and NCC-S1M cell lines were selected for OPN knockdown because the AGS cell line was one of the gastric cancer cell lines showing a relatively high expression of OPN based on western blot analysis (data not shown), and the NCC-S1M line showed a considerably increased OPN expression compared with mouse normal gastric epithelium (Supplementary Figure S1B). Cells were treated by $2 \mu \mathrm{g} / \mathrm{ml}$ puromycin at $48 \mathrm{~h}$ after viral transduction and were selected for 3 days. The knockdown efficiency was determined by quantitative real-time RT-PCR (QRT-PCR) and western blotting (Supplementary Figure S1).

\section{In Vitro Migration Assay}

We performed a modified Boyden chamber migration assay to measure migration of mouse peritoneal macrophages, THP-1 cells, and RAW264.7 cells using transwell 24-well inserts with an $8 \mu \mathrm{m}$ porous membrane (353097, BD Biosciences). Either medium containing $H P$ filtrates or the CM was added to the 24-well lower chamber (354578, BD Biosciences). HP filtrates were prepared as previously described. ${ }^{20}$ The cells were placed on 24-well inserts at 
$1 \times 10^{5}$ cells per well in serum-free RPMI-1640 media. After $16 \mathrm{~h}$, mouse peritoneal macrophages and RAW264.7 cells had migrated toward the insert chamber and attached on the lower side of the membrane. The insert was fixed with $10 \%$ formalin for $15 \mathrm{~min}$ and soaked in hematoxylin for $1 \mathrm{~min}$. Membrane was cut from the insert and placed on a glass slide and mounted under a coverslip with Permount (Fisher Scientific, Waltham, MA, USA). The number of migrated cells was averaged by counting three high-power fields $(\times 200)$. As THP-1 cells had migrated completely through the membrane, total media from the lower chamber were collected and the number of migrated cells was counted using Trypan blue exclusion assay.

\section{Quantitative Real-Time RT-PCR}

Total RNA from both stomach tissues and cell lines was extracted by RNeasy Plus Mini kit (Qiagen) according to the manufacturer's instructions. From each sample, $0.1 \mu \mathrm{g}$ of total RNA was reverse-transcribed using the QuantiTect Reverse Transcription kit (Qiagen) and analyzed by real-time PCR using the Rotor-Gene SYBR Green PCR kit (Qiagen) with specific primers. Target genes were amplified and quantified using the Rotor-Gene Q and manufacturer's software (Qiagen). The amount of target gene was calculated using mRNA encoding $\beta$-actin and GAPDH as a housekeeping gene. QRT-PCR primers for mouse genes were F: $5^{\prime}$-GGAGAACC AAGCAACGACAA- $3^{\prime}$ and R: $5^{\prime}$-TGGGGAACTCTGCAGACT CA-3' for $I l 1 b$, F: 5'-AGCCCCCACTCTGACCCCTTTAC-3' and R: 5'-TGTCCCAGCATCTTGTGTTTCT-3' for Tnf, F: 5'-AGCGGCTGACTGAACTCAGATTGTAG-3' and R: 5'-GT CACAGTTTTCAGCTGTATAGGG-3' for Ifng, and F: 5'-CA GGAGATGGCCACTGCCGCA-3' R: $5^{\prime}$-TCCTTCTGCATCC TGTCAGCA-3' for Actb. QRT-PCR primers for human genes were F: 5'-CCAGTGAAATGATGGCTTATTAC- $3^{\prime}$ and R: 5'-CTGTAGTGGTGGTGGTCGGAGATT-3' for IL1B, F: 5'-GCTTGTTCCTCAGCCTCTTCT-3' and R: 5'-GGTTTGC TACAACATGGGCTA-3' for TNF, and F: 5'-GAGTCAACGG ATTTGGTCG-3' R: 5'-TGGAATCATATTGGAACATGTA AAC-3' for GAPDH.

\section{Western Blotting (WB)}

Both mouse stomach tissues and cell lines were lysed with T-PER Tissue Protein Extraction Reagent (Thermo Fisher Scientific, Hudson, NH, USA) supplemented with protease inhibitor (P3100-001, GenDEPOT, Barker, TX, USA) and phosphatase inhibitor (P3200-001, GenDEPOT) according to the manufacturer's instructions. Protein samples were mixed with $4 \times$ Laemmli sample buffer and boiled for $5 \mathrm{~min}$ at $100^{\circ} \mathrm{C}$. Equal amounts of protein were separated on SDS-polyacrylamide gel and transferred onto nitrocellulose membrane by electrophoresis and blotting apparatus (Bio-Rad, Hercules, CA, USA). The proteins were probed with the primary antibodies and horseradish peroxidase (HRP)-conjugated secondary antibodies at the recommended dilutions. The following primary antibodies were used in this study; goat polyclonal anti-mouse OPN antibody (1:100; AF808, R\&D Systems), goat polyclonal anti-human OPN antibody (1:100; AF808, R\&D Systems), rabbit polyclonal anti-p21antibody (1:1000; sc-397, Santa Cruz Biotechnology, Santa Cruz, CA, USA), rabbit polyclonal anti-Cyclin E antibody (1:1000; 07-687, Merck Millipore, Billerica, MA, USA), rabbit polyclonal anti phospho-Akt1/2/3 (Thr 308) antibody (1:1000; sc-16646-R, Santa Cruz Biotechnology), rabbit polyclonal anti-Akt $1 / 2 / 3$ antibody (1:1000; sc-8312, Santa Cruz Biotechnology), mouse monoclonal anti phosphop44/42 MAPK (pErk1/2) antibody (1:1000; 9106, Cell Signaling Technology, Beverly, MA, USA), rabbit polyclonal anti p44/42 MAPK (Erk1/2) antibody (1:1000; sc-292838, Santa Cruz Biotechnology), and mouse monoclonal anti$\beta$-actin antibody (1:1000; sc-47778, Santa Cruz Biotechnology). Immunodetection was performed by using an enhanced chemiluminescence (ECL) detection kit (Thermo Fisher Scientific).

\section{Statistical Analysis}

All data are expressed as means \pm s.e.m. Statistical analyses were performed by GraphPad Prism 4 (version 4.0; GraphPad Software, San Diego, CA, USA). The data were analyzed using unpaired two-tailed Student's $t$-test. $P$-values of ${ }^{<} 0.05$ were considered statistically significant.

\section{Results}

\section{Attenuated Inflammation in HP-Infected OPN KO Mice}

We confirmed whether $H P$ successfully infected WT and OPN KO mice using TaqMan real-time PCR amplification of the $16 \mathrm{~S}$ rDNA gene of HP SS1. ${ }^{17} \mathrm{HP}$ burden between OPN $\mathrm{KO}$ and WT was not statistically different, indicating $H P$ burden is not directly affected by OPN gene itself (Figure 1a). We then compared the degree of inflammatory changes between two groups at 8 and 16 weeks after $H P$ infection as previously described. ${ }^{19}$ WT mice exhibited gastritis displaying multifocal infiltration of moderate numbers of neutrophils, macrophages, and lymphocytes in the lamina propria and submucosa at 8 weeks (Figures $1 \mathrm{~b}$ and c). In WT mice at 16 weeks, the inflammatory response expanded and occasionally infiltrated below the submucosa. Meanwhile, OPN KO mice showed an attenuation of the inflammation compared with WT mice at both 8 and 16 weeks (Figure 1c), as confirmed by histological grading (Figure 1b). The numbers of infiltrating macrophages (F4/80-positive cells), T cells (CD3-positive cells), and neutrophils (polymorphonuclear cells) in $H P$-infected OPN KO mice were significantly decreased compared with that of HP-infected WT mice (Figures 1d and e). The reduction in infiltrating macrophages was especially prominent in OPN KO mice infected with $H P(P<0.0001)$. These results demonstrate that OPN deficiency reduces gastric inflammatory response to $H P$ infection. In addition, WT mice occasionally showed epithelial defects such as slightly dilated glands and mild surface erosion at both 8 and 16 weeks, but these lesions were either not 
a

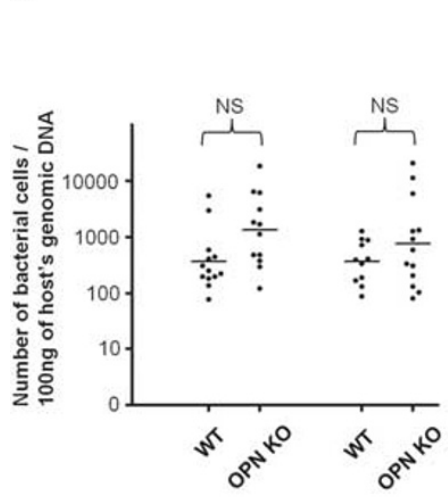

b

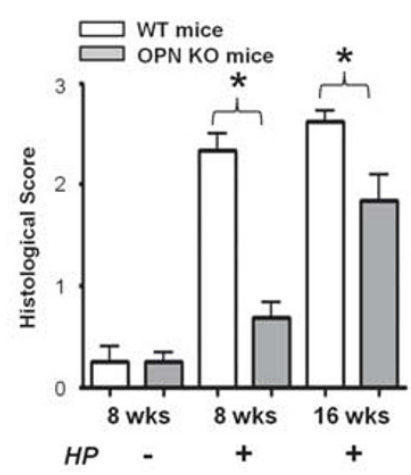

c

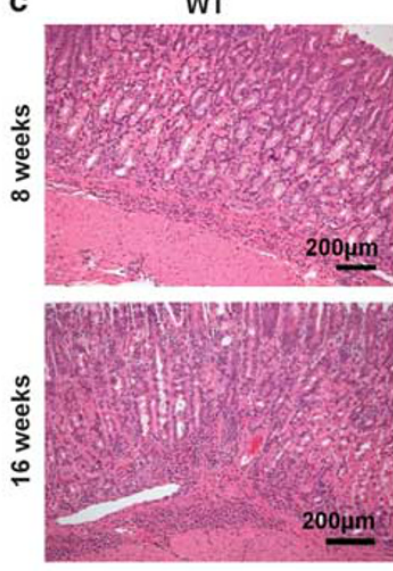

e
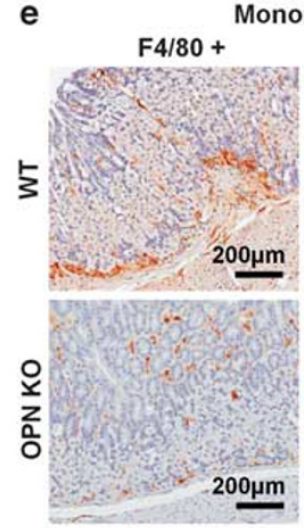

$\mathrm{CD} 3+$

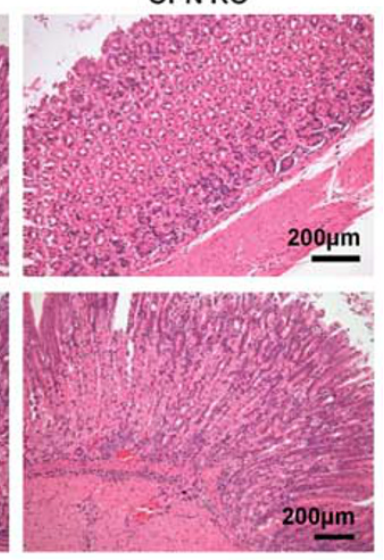

Polymorphonuclear
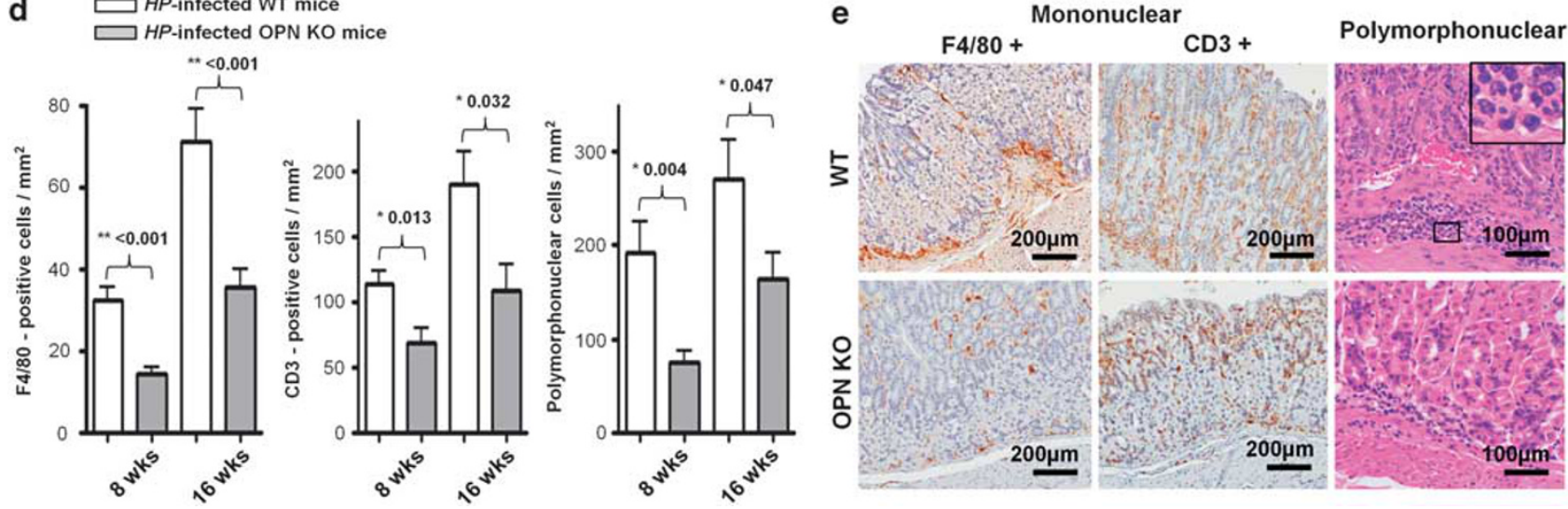

Figure 1 Histopathological and immunohistochemical findings of HP-induced gastritis in WT and OPN KO mice. (a) Assessment for bacterial colonization in HP-infected stomach tissues. (b) Histopathological scoring for gastritis. Results are presented as mean \pm s.e.m. ( $n=10)$. (c) Representative H\&E images of gastric tissues at both 8 and 16 weeks after infection. (d) Quantitation of F4/80, CD3-positive cells, and polymorphonuclear cells at 8 and 16 weeks after infection. Results are presented as mean \pm s.e.m. $(n=10)$. (e) Representative immunohistochemical images of inflammatory cells infiltrated in stomach tissues. $P$-values $<0.05$ are designated with asterisks.

detected or mild in OPN KO mice (Supplementary Figure S2). $H P$-infected WT mice also showed 1.5- to 2-fold increases in the isthmus length compared with uninfected WT mice (Supplementary Figure S2), but the hyperplastic change was significantly reduced in OPN KO mice at 16 weeks after $H P$ infection. TFF2-expressing metaplasia (SPEM) in gastric mucosa was observed in neither WT nor OPN KO mice until 16 weeks after HP infection (Supplementary Figure S3).

\section{Gastric OPN Expression Is Upregulated After HP Infection, and Both Gastric Epithelial Cells and Macrophages Are the Source of OPN}

We next examined whether $H P$ infection upregulated OPN expression in murine gastric tissues. In noninfected WT gastric tissues, WB analysis detected minimal OPN expression (Figure 2a) and IHC showed weak and scattered random OPN expression in the gastric glands (Figure 2bi). As expected, OPN expression was not detected in OPN KO mice (Figure 2bi). In contrast, WB analysis showed mild increase of OPN in WT mice at 8 weeks after HP infection (Figure 2a).
IHC revealed moderate expression of OPN in a small population of infiltrating inflammatory cells, whereas the increased OPN expression was not prominent in the gastric epithelium (Figure 2bii). Double immunofluorescence (IF) staining for OPN and F4/80 showed that inflammatory cells expressing OPN were mainly macrophages (Figure $2 \mathrm{c}$ ). These results indicate that source of gastric OPN expression at earlystage $H P$ infection is infiltrating macrophages rather than gastric epithelium. However, there was a marked upregulation of gastric OPN in WT mice at 16 weeks after HP infection compared with 8 week on WB (Figure 2a). Immunohistochemically, in contrast to 8-week response, the major source of OPN expression is gastric glandular epithelial cells rather than infiltrating macrophages (Figure 2bii). These results demonstrate a time-dependent increase in gastric OPN expression according to disease progression and implicate OPN in gastric epithelial responses to the infection.

We performed in vitro studies to confirm that the increases in OPN expression in response to $H P$ infection were attributable to both gastric epithelial cells and macrophages. 
a

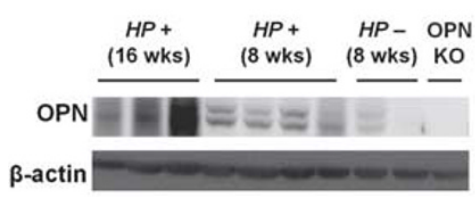

C
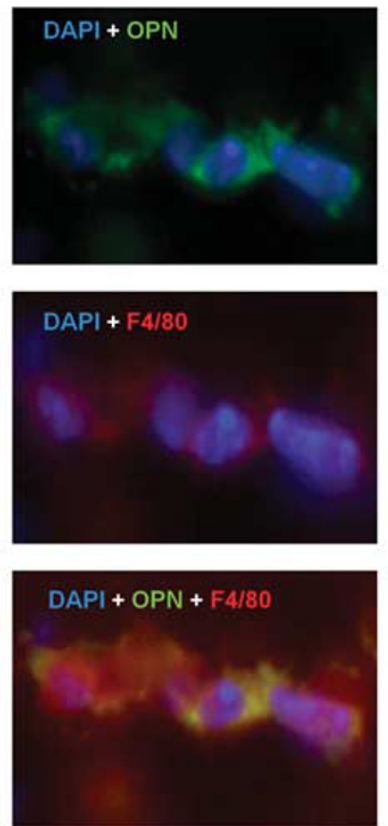

b

(i)
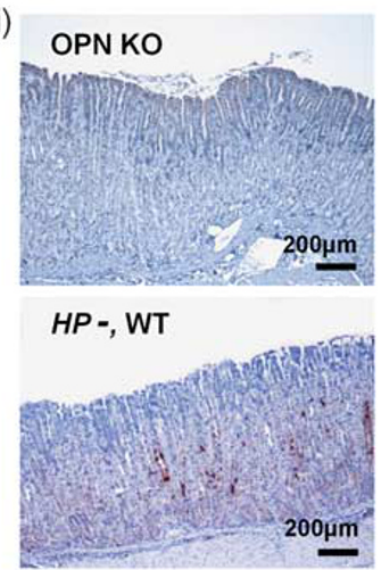
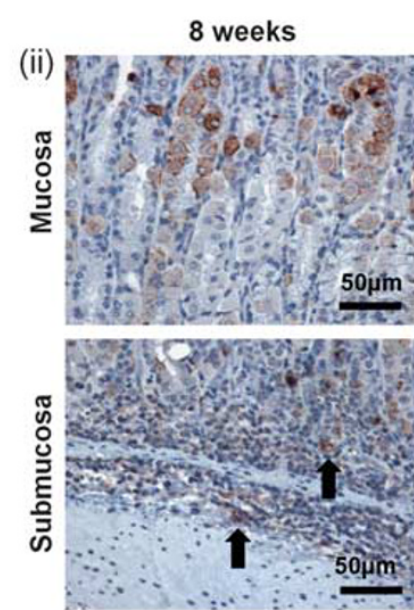
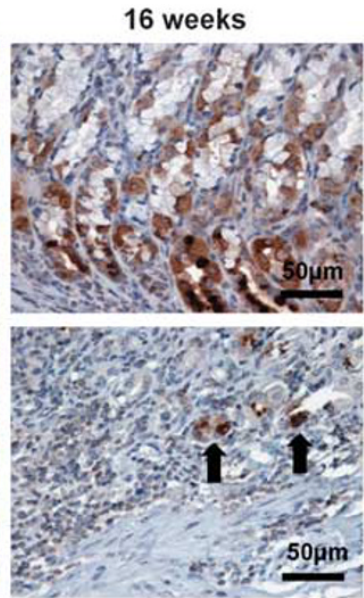
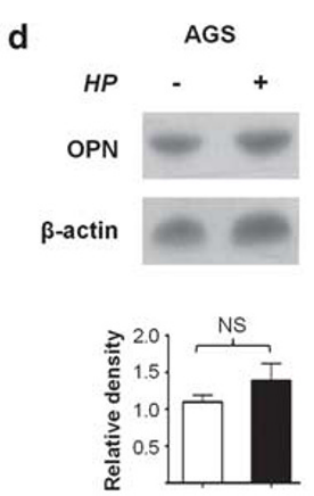
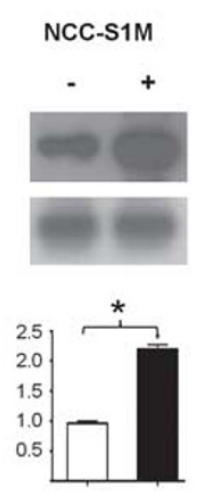
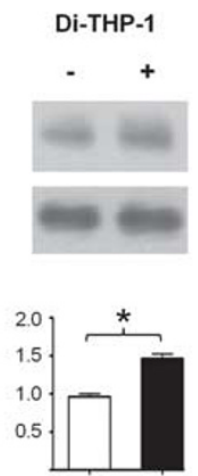
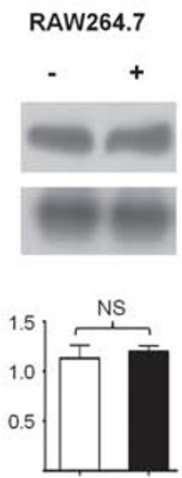

Peritoneal macrophage
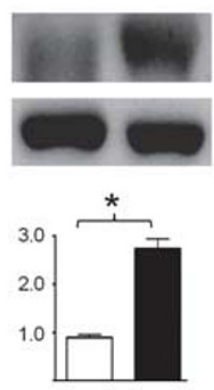

Figure 2 OPN expression in HP-infected stomach tissues of WT mice and various cell lines. (a) Western blot analysis for OPN of HP-infected or noninfected WT mice. (b) Representative immunohistochemistry for OPN in HP-infected WT stomach tissues. (b (i)) OPN KO stomach tissue and noninfected WT stomach tissue. (b (ii)) OPN expression was elevated in gastric epithelial cells of mucosal layer at 16 weeks after HP infection compared with 8 weeks. Inflammatory cells in submucosal layer frequently showed OPN immunoreactivity (arrow) during HP infection. (c) Double immunofluorescence for F4/80 and OPN. OPN was expressed in F4/80-positive cells. Original magnification $\times 1000$. (d) OPN expression in various cell lines and peritoneal macrophages at $24 \mathrm{~h}$ after HP exposure. $P$-values $<0.05$ are designated with asterisks.

WB showed that OPN expression did not change in AGS cells at $24 \mathrm{~h}$ after co-culture with $\mathrm{HP}(20 \mathrm{MOI}$; Figure $2 \mathrm{~d})$. On the other hand, OPN expression was mildly up-regulated in Di-THP-1 at $24 \mathrm{~h}$ after HP co-culture (Figure 2d). In contrast, over twofold increase of OPN expression was observed in NCC-S1M cells, but not in RAW 264.7 cells, at $24 \mathrm{~h}$ after HP co-culture (Figure 2d). Peritoneal macrophages isolated from WT mice showed over twofold increase of OPN expression after $H P$ exposure (Figure $2 \mathrm{~d}$ ). These results suggest that increased expression of OPN in the HP-infected stomach originate from both epithelial cells and macrophages in a context-dependent manner.

\section{OPN Deficiency Downregulates Major Proinflammatory Cytokines Induced by HP Infection}

We investigated whether OPN deficiency influenced the expression of cytokines in $H P$-infected stomach tissues. The QRT-PCR analysis revealed decreased mRNA expression of
IL- $1 \beta$, TNF- $\alpha$, and IFN- $\gamma$ in OPN KO mice compared with WT mice at 8 weeks after HP infection (Figure 3a). By 16 weeks after HP infection, although IL- $1 \beta$ and TNF- $\alpha$ mRNA levels were not significantly different between WT and OPN KO mice, reduced expression of IFN- $\gamma$ was observed in OPN KO mice compared with WT mice (Figure 3a).

We further performed in vitro studies to investigate which types of cells might account for the altered expression of these cytokines in HP-infected OPN KO mice. Both OPN KD AGS and OPN KD NCC-S1M cells co-cultured with HP showed reduced IL- $1 \beta$ and TNF- $\alpha$ mRNA expression compared with shRNA control cells (Figures $3 \mathrm{~b}$ and c). Like HP-stimulated OPN KD gastric cancer cells, OPN KD Di-THP-1 cells and OPN KO peritoneal macrophages showed reduced expression of these cytokines compared with shRNA control Di-THP-1 cells and WT peritoneal macrophages, respectively, following co-culture with $H P$ (Figures $3 \mathrm{~d}$ and e). Taken together, these observations indicate that the altered expression of these 


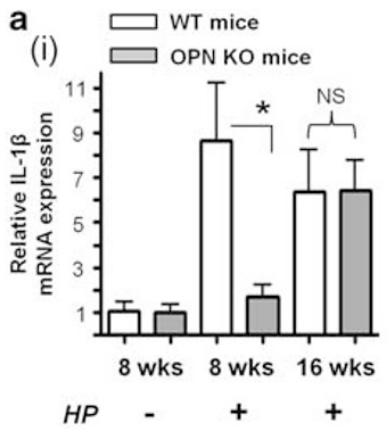

(ii)

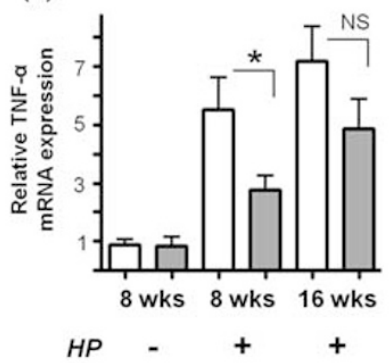

(iii)

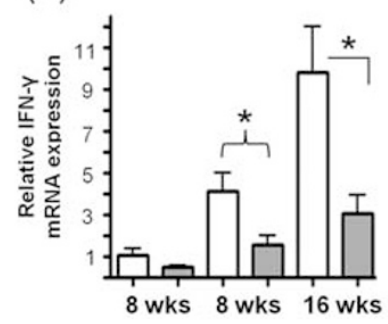

b (i) $\begin{aligned} & \text { shControl AGS } \\ & \text { OPN KD AGS }\end{aligned}$

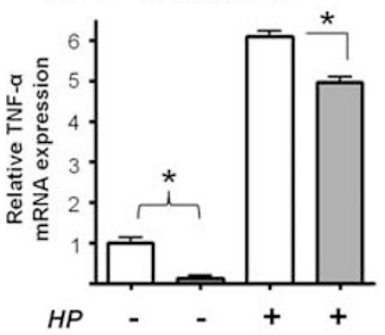

d

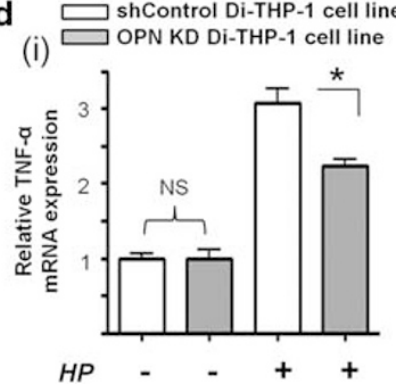

(ii)

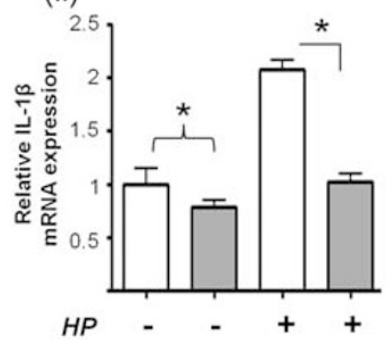

(ii)

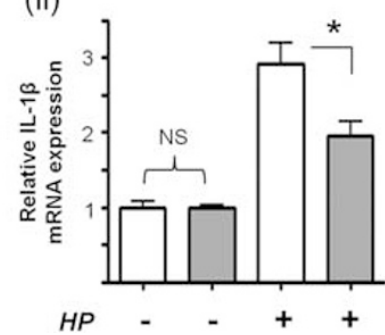

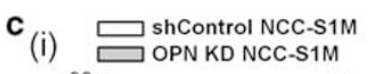
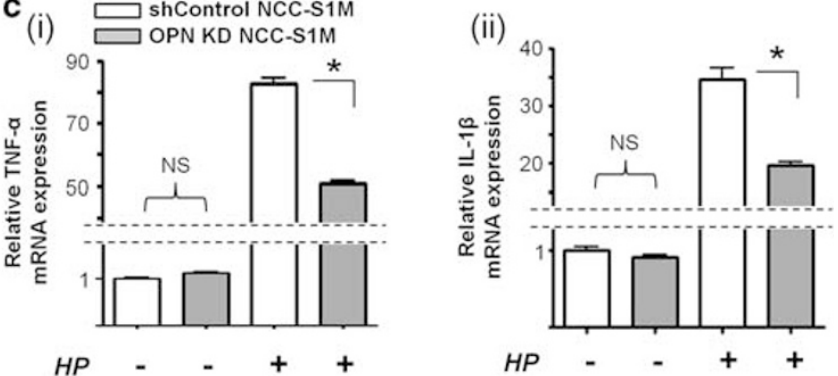

e $\square$ WT peritoneal macrophage (i) $\square$ OPN KD peritoneal macrophage

(ii)
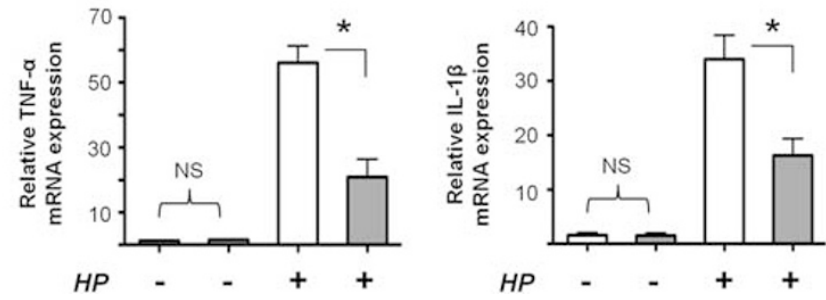

Figure 3 Relative mRNA expression levels of proinflammatory cytokines in HP-infected OPN KO mice and OPN KD cell lines. (a) Expression level of (a (i)) IL-1 $\beta$, (a (ii)) TNF- $\alpha$, and (a (iii)) IFN- $\gamma$ in stomach tissues of HP-infected WT and OPN KO mice. Results are presented as mean $\pm s$.e.m. ( $n=6$ ). (b-d) Effect of OPN knockdown or knockout on (i) TNF- $\alpha$ and (ii) IL-1 $\beta$ expression in (b) AGS cells, (c) NCC-S1M cells, (d) Di-THP-1 cells, and (e) peritoneal macrophages at $24 \mathrm{~h}$ after HP exposure. $P$-values $<0.05$ are designated with asterisks.

cytokines in response to $H P$ infection might be attributable to a change in the production of these cytokines in both gastric epithelial cells and infiltrating macrophages.

\section{OPN Deficiency Suppresses the Migration of Macrophages in Response to HP Infection}

Because HP-infected OPN KO mice showed a significant reduction in macrophage infiltration, we investigated the role of OPN in macrophage migration in response to HP infection using a modified Boyden chamber. Peritoneal macrophages from OPN KO mice showed reduced migration toward media containing HP infiltrates compared with those from WT mice (Figure 4a). OPN KD THP-1 cells also showed reduced migratory activity toward media containing $H P$ infiltrates compared with shRNA control THP-1 cells (Figure 4b). In addition, we tested the effects of altered cytokine expression in $\mathrm{HP}$-infected OPN KD AGS cells (Figure $3 \mathrm{~b}$ ) on the migration of inflammatory cells. CM harvested from
HP-co-cultured OPN KD AGS and OPN KD NCC-S1M cells decreased the migratory behavior of THP-1 and RAW 264.7 cells compared with CM from HP-co-cultured shRNA control cells (Figures $4 \mathrm{c}$ and d). These results show that the reduced infiltration of macrophages in the stomach of HP-infected OPN KO mice is because of reduction in the migratory activity of macrophages as well as decreased production by gastric epithelial cells of soluble factors that attract macrophages.

\section{OPN Deficiency Inhibits the Proliferation of Gastric Epithelial Cells in Response to HP Infection}

$H P$ infection can modulate gastric epithelial cell proliferation and apoptosis contributing to the development of gastric cancer. ${ }^{21,22}$ Based on the low degree of mucosal hyperplasia in OPN KO mice compared with WT mice after HP infection (Supplementary Figure S2), we performed IHC for Ki-67 on stomach tissues of $H P$-infected mice to investigate the impact 

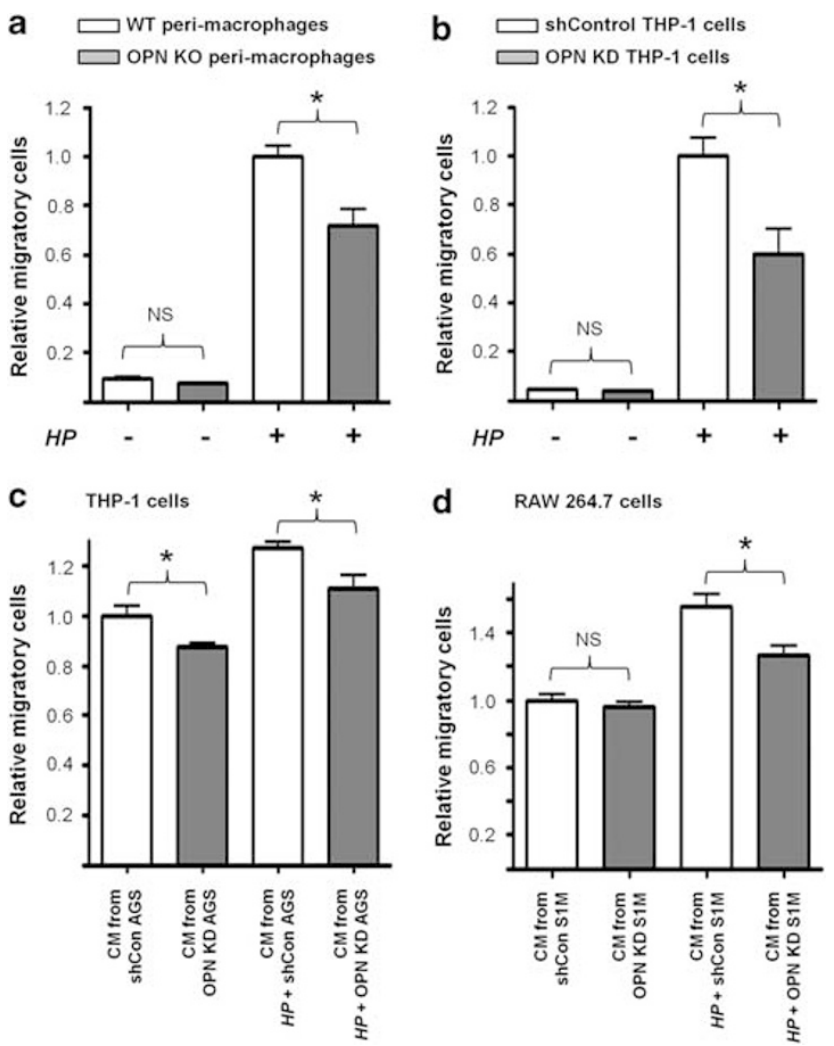

Figure 4 Effect of OPN on migratory activity of inflammatory cells in response to HP. Migratory activities of (a) OPN KO mouse peritoneal macrophages and (b) OPN KD THP-1 cells toward HP infiltrate-containing media. Migratory activities of (c) THP-1 cells and (d) RAW 264.7 cells toward CMs harvested from OPN KD AGS cells or S1M cells co-cultured with $H P$. $P$-values $<0.05$ are designated with asterisks.

of the lack of OPN on gastric epithelial proliferation in response to $H P$ infection. We found out that the number of Ki-67-positive gastric epithelial cells in $H P$-infected WT mice at 8 and 16 weeks after $H P$ infection was significantly higher than that of uninfected WT mice (Figures 5a and b). Notably, $\mathrm{HP}$-infected OPN KO mice showed lower numbers of proliferative gastric epithelial cells than $H P$-infected WT mice at both 8 and 16 weeks after $H P$ infection (Figures $5 \mathrm{a}$ and b). In addition, IHC for PNCA, a specific marker of cell division, also demonstrated that $H P$-infected OPN KO mice showed a lower number of PCNA-positive gastric epithelial cells than WT mice at 8 weeks after $H P$ infection (Supplementary Figure S4). These results indicate that the lack of OPN led to decreased proliferation of gastric epithelial cells in response to $H P$ infection.

Similar results were obtained in vitro, where OPN KD AGS cells showed reduced cell viability compared with shRNA control AGS cells at $24 \mathrm{~h}$ after HP co-culture (Figure $5 \mathrm{ci}$ ). This result might be attributable to suppression of the G1/S cell-cycle checkpoint in OPN KD AGS cells (Figure 5cii) that was accompanied by increased induction of cyclin-dependent kinase inhibitor p21 and decreased expression of Cyclin E
(Figure 5ciii). OPN KD S1M cells also exhibited reduced cell viability compared with shRNA control cells after $H P$ co-culture for $24 \mathrm{~h}$, showing results similar to those of the cell cycle analysis and molecular changes observed in OPN KD AGS cells (Figure 5d). Thus, these results demonstrate that OPN is involved in cycle regulation of gastric epithelial cells by controlling the G1/S transition during $H P$ infection.

We investigated the effects of OPN on IL- $1 \beta$ and TNF- $\alpha$ induced gastric epithelial proliferation, because IL- $1 \beta$ and TNF- $\alpha$ were important mediators that could be responsible for gastric epithelial changes in response to $H P$ infection. ${ }^{11,23,24}$ OPN KD AGS and NCC-S1M cells showed decreased cell proliferation compared with shRNA control cells following exposure to IL- $1 \beta$ and TNF- $\alpha$ for $24 \mathrm{~h}$ (Figure 6a). In addition, compared with shRNA control cells, OPN KD AGS and S1M cells showed an attenuation of the increase in the mitogen-activated protein kinase (MAPK)related molecule, phosphorylated Akt, and Erk following exposure to IL- $1 \beta$ and TNF- $\alpha$ treatment (Figures $6 \mathrm{~b}-\mathrm{e}$ ). The induction of MAPK-related molecules induced by $H P$ infection also decreased in OPN KO mice than in WT mice (Figure 6f).

\section{Discussion}

In this study, we investigated the roles and mechanism of OPN in HP-induced gastritis in mice. The degree of inflammation of OPN KO mice was weak compared with that of WT mice, with a significant reduction in infiltrating macrophages and the expression of IL- $1 \beta$, TNF- $\alpha$, and IFN- $\gamma$. Similar with these results, mRNA expression of these proinflammatory cytokines was reduced in OPN KD gastric cancer cell lines exposed to HP, and the CM from these cells had a decreased capacity on the migration of monocytic and macrophage-like cell lines. Furthermore, HP-infected OPN $\mathrm{KO}$ mice showed a lower number of proliferative gastric epithelial cells than WT mice, in association with a reduction in MAPK pathway activation. OPN KD gastric cancer cell lines also showed the suppression of the G1/S cell cycle after $H P$ co-culture and reduced MAPK activation after IL- $1 \beta$ and TNF- $\alpha$ treatment. To our knowledge, this is the first study to explore the functional roles of OPN during HP infection.

The immune response of the host during $H P$ infection is considered to be a key event in the development of gastric cancer. It has consistently been reported that gastric mucosal levels of the proinflammatory cytokines IL-1 $\beta$, IL-6, IL-8, IFN- $\gamma$, and TNF- $\alpha$ increased in $H P$-infected individuals. ${ }^{25,26}$ Notably, there is considerable evidence that elevated expression of IL- $1 \beta$, IFN- $\gamma$, and TNF- $\alpha$ are associated with chronic gastritis and gastric cancer development following $H P$ infection. ${ }^{7,11,25,27,28}$ Our study revealed that OPN expression was elevated in response to HP infection, and OPN depletion decreased production of these cytokines in both gastric epithelial cells and macrophages during HP infection. These results suggest that OPN is an important mediator of proinflammatory cytokine production during $H P$ infection. 
a

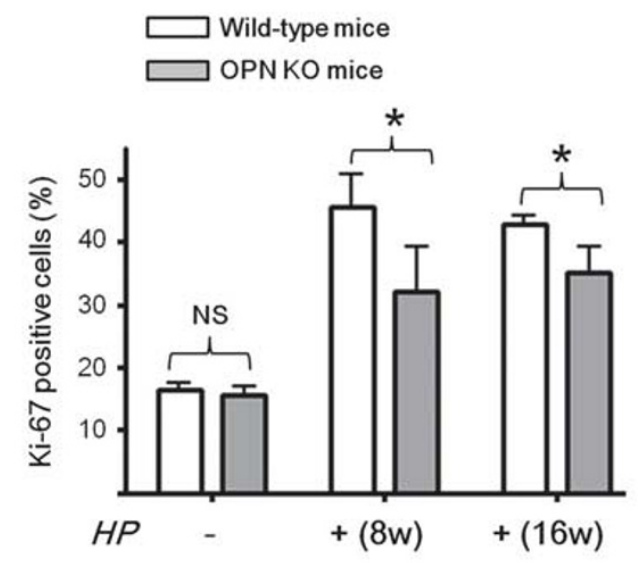

b

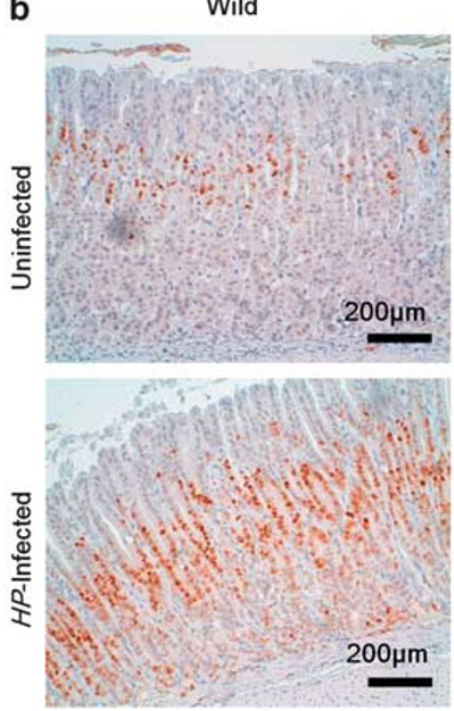

OPNKO

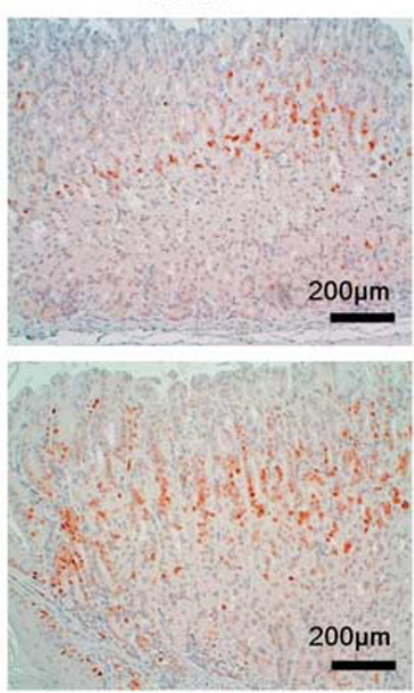

C (i)

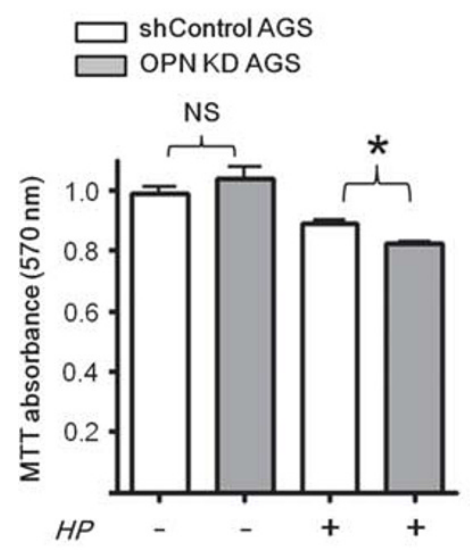

d

(i) $\square$ shControl S1M

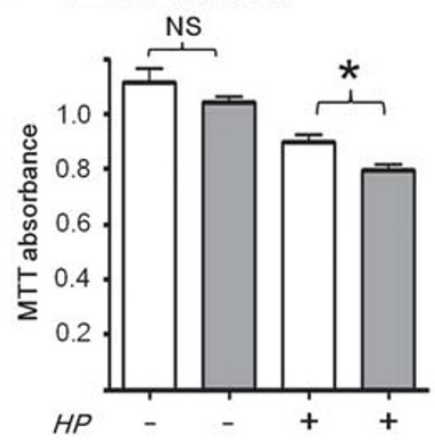

(ii)
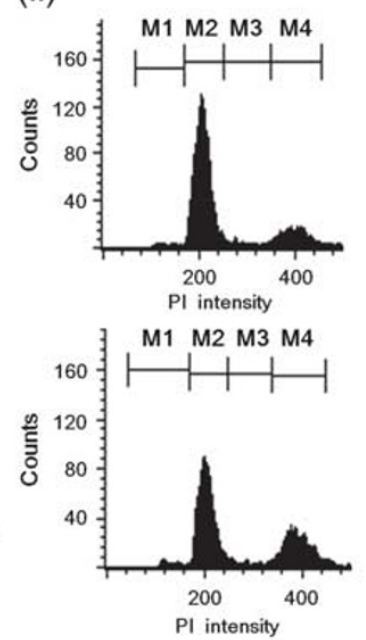

(ii)

\begin{tabular}{cc}
\multicolumn{2}{c}{ shCON S1M } \\
\hline Marker & $\%$ \\
\hline SubG1 & 3.21 \\
G1 & 45.44 \\
S & 32.64 \\
G2/M & 17.71 \\
\hline
\end{tabular}
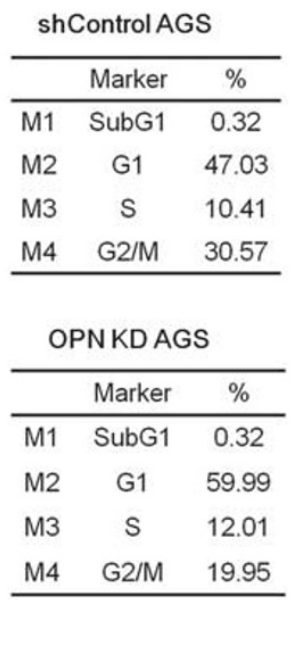

(iii)

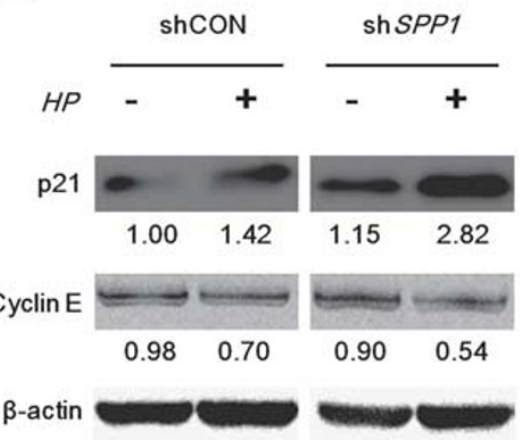

\begin{tabular}{cc}
\multicolumn{2}{c}{ OPN KD S1M } \\
\hline Marker & $\%$ \\
\hline SubG1 & 3.87 \\
G1 & 50.92 \\
S & 28.27 \\
G2/M & 16.23 \\
\hline
\end{tabular}

(iii)
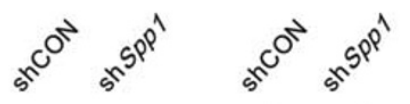

$H P$

p21
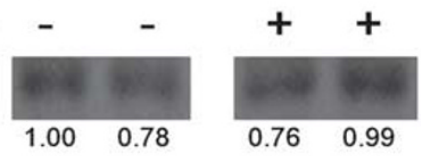

Cylcin E
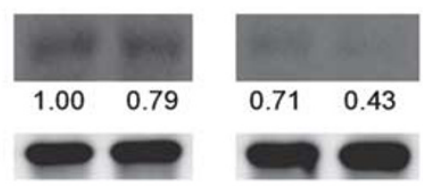

Figure 5 Effect of OPN on gastric epithelial proliferation during HP infection. (a) Quantitation of Ki-67-positive cells in stomach tissues of WT and OPN KO mice. Results are presented as mean \pm s.e.m. $(n=10)$. (b) Representative images of immunohistochemistry for Ki- 67 in stomach tissues at 8 weeks after infection. (c) Effect of OPN knockdown in AGS cells on (ci) cell viability and (c (ii)) cell cycle after HP co-culture. (c (iii)) Western blotting for cell cycle regulatory proteins in HP-infected OPN KD AGS cells. (d) Effect of OPN knockdown in S1M cells on (d (i)) cell viability, (d (ii)) cell cycle, and (d (iii)) cell cycle regulatory proteins after HP co-culture. $P$-values $<0.05$ are designated with asterisks. 
a

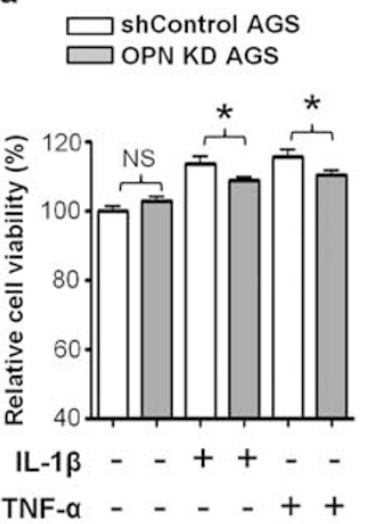

$\square$ shControl NCC-S1M OPN KD NCC-S1M

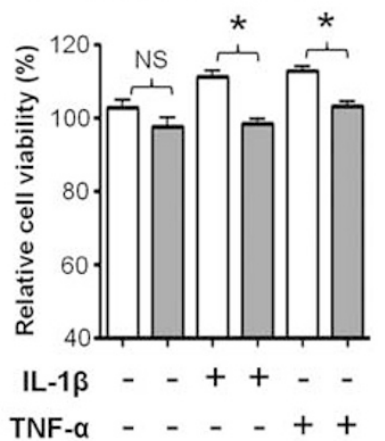

b
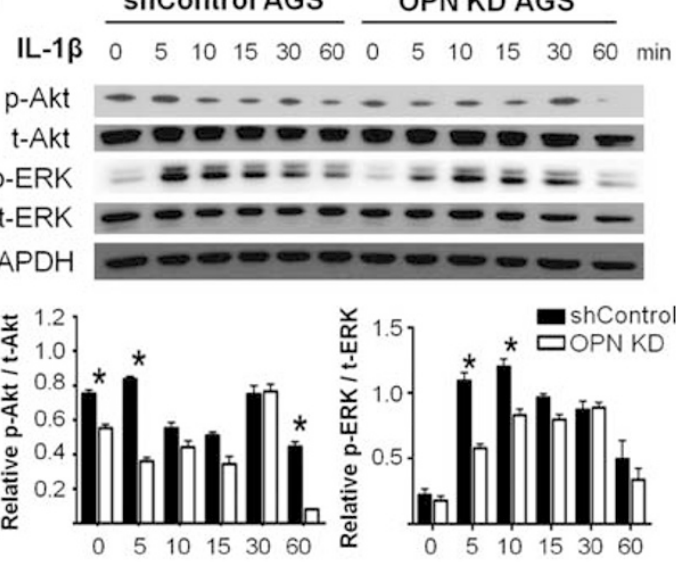

d
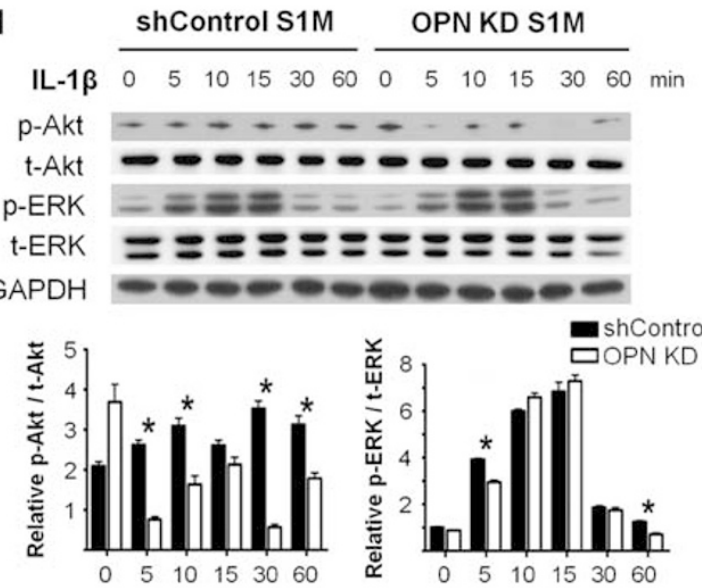

f

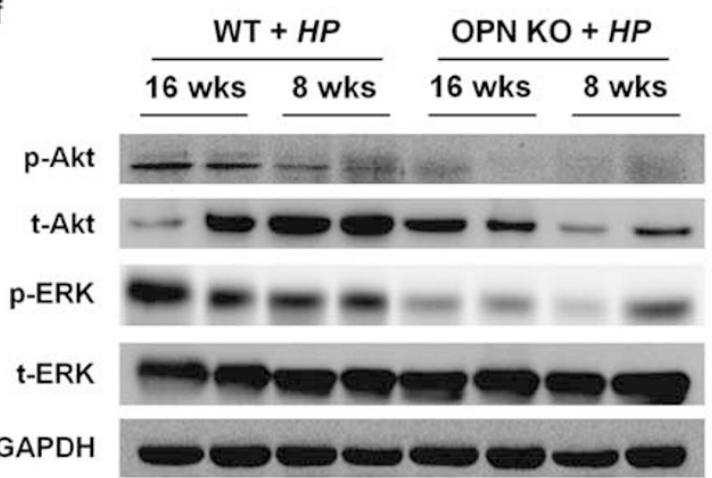

e
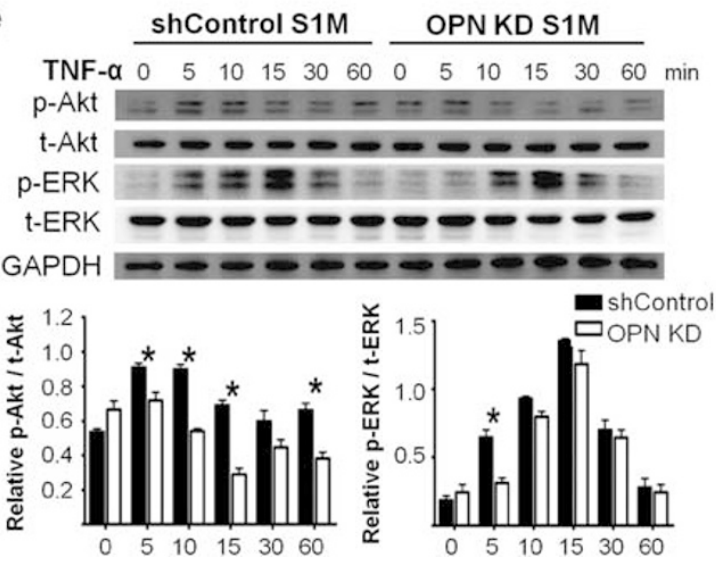

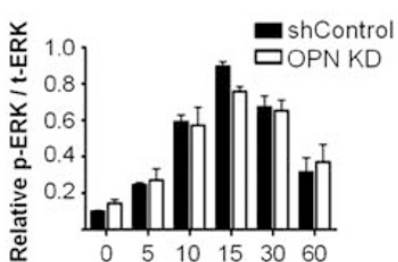

Figure 6 Effect of OPN on the activation of MAPK pathway in gastric cancer cells and mouse gastric tissues during HP infection. (a) MTT assays for cell viability at $24 \mathrm{~h}$ after IL-1 $\beta$ or TNF- $\alpha$ treatment in OPN KD gastric cancer cells. MAPK-related protein expression in OPN KD AGS cells treated with (b) IL-1 $\beta$ and (c) TNF- $\alpha$ at the respective time points. Expression levels of MAPK-related proteins in OPN KD S1M cell treated with (d) IL-1 $\beta$ and (e) TNF- $a$. (f) MAPK-related protein expression in OPN KO mouse gastric tissues infected with HP. Results are presented as mean \pm s.e.m. $(n=2)$. ${ }^{*} P<0.05$ versus shControl-transfected cell. $\mathrm{p}$-Akt, phosphorylated Akt; $\mathrm{t}$-Akt, total Akt; $\mathrm{p}$-ERK, phosphorylated ERK; $\mathrm{t}$-ERK, total ERK.

Previous studies have focused on the role of OPN in inflammatory cells and have reported that OPN induces the expression of these cytokines in inflammatory cells, such as macrophages, dendritic cells, and $\mathrm{T}$ cells. ${ }^{29,30}$ However, the effect of OPN on epithelial cell in the development of inflammatory conditions has not yet been studied. For the first time, we demonstrate the involvement of OPN in the production of proinflammatory cytokines by gastric epithelial cells in the immune response against $H P$ in addition to inflammatory cells. 
Macrophage infiltration is a characteristic feature of $H P$ infection that may promote progression or resolution of chronic gastritis following HP infection. ${ }^{31}$ Indeed, we found that the number of infiltrated macrophages was positively correlated with the grade of gastritis in HP-infected WT mice (data not shown). Importantly, OPN KO mice showed a significant reduction in the number of infiltrating macrophages following $H P$ infection compared with WT mice. This reduction could result from the downregulated expression of chemoattractants, such as IL- $1 \beta$, IFN- $\gamma$, and TNF- $\alpha$, in OPN-deficient gastric epithelial and inflammatory cells as well as the decrease in the migratory ability of monocytes and macrophages lacking OPN. The demonstration that CM from $H P$-infected OPN KD gastric cancer cell lines had a reduced ability to recruit monocytic and macrophage-like cells provides support for this hypothesis.

$H P$ infection enhances the proliferative activity of gastric epithelial cells..$^{9,22,32,33}$ The gastric epithelial cell proliferation results from both the direct effects of $H P$ pathogenic factor, such as $\mathrm{CagA},{ }^{34}$ and the indirect effects of inflammatory environment enriched with cytokines, such as TNF- $\alpha$ and IL- $1 \beta$, induced by $H P$ infection ${ }^{24,35}$ that could activate the MAPK cascades. As expected, HP-infected WT mice showed elevated gastric epithelial proliferation in association with enhanced MAPK pathway activation. In contrast, epithelial proliferation and MAPK pathway activation induced by $H P$ infection was suppressed in OPN KO mice, suggesting that OPN is associated with MAPK-mediated epithelial proliferative activity in response to $H P$ infection. This conclusion is supported by the demonstration that $H P$-stimulated OPN KD gastric cancer cell lines showed the suppression of the G1/S cell cycle transition, of which the MAPK pathway is a master regulator. ${ }^{36}$ In addition to gastric epithelial proliferation via a direct interaction between $H P$ and gastric epithelial cells, our results also showed that IL- $1 \beta$ and TNF- $\alpha$ increased the proliferation of gastric cancer cells, in association with upregulated expression of MAPK pathway-related proteins. These effects were attenuated in OPN KD gastric cancer cells. Taken together, our findings suggest that OPN depletion suppresses $H P$-induced gastric epithelial proliferation via suppression of the MAPK pathway.

Our study shows that OPN deficiency suppressed $H P$-induced gastritis and gastric epithelial proliferation, and we focused on the roles of OPN in gastric epithelial cells and macrophages during HP infection. Although OPN is not essential for normal mouse development and physiology, ${ }^{37}$ it is a multifunctional protein that is upregulated in various types of cells in a variety of diseases, where it mediates diverse cellular functions including regulating the inflammatory response of macrophages, $\mathrm{T}$ cells, and dendritic cells. ${ }^{29}$ However, the OPN KO mice used in this study were deficient in the OPN expression of whole tissues and cells. In this respect, it is necessary to consider the possibility that OPN in other stromal cells, such as neutrophils, dendritic cells, and lymphocytes, was involved in the HP-induced gastritis.
Actually, as shown in Figure 1d, the numbers of infiltrating $\mathrm{T}$ cells and neutrophils in the HP-infected OPN KO mice were significantly decreased compared with those of the HP-infected WT mice. In addition, OPN could be involved in monocyte migration and differentiation into macrophages during infection, because OPN was strongly induced when human monocytic cell lines were treated with PMA. ${ }^{38}$ In addition, other activated or inactivated pathways in OPN $\mathrm{KO}$ mice during mouse development might be involved in the immunosuppressive effect to $H P$-induced gastritis. To specify the role of OPN and clearly elucidate which types of cells play important roles in $H P$-induced gastritis, further studies using cell- or tissue-specific OPN knockout mice may be helpful.

In summary, the present study has demonstrated that OPN depletion decreased inflammation and gastric epithelial proliferation in $H P$-infected mice, establishing the importance of OPN as a mediator of the gastritis induced by $H P$ infection. In addition, our results suggest that targeting OPN might be an appropriate therapeutic strategy for limiting inflammation and mucosal pathology during HP infection.

Supplementary Information accompanies the paper on the Laboratory
Investigation website (http://www.laboratoryinvestigation.org)

\section{ACKNOWLEDGMENTS}

The work was supported by Research Institute for Veterinary Science, College of Veterinary Medicine, by National Cancer Center Grants 1410850 and by the Proteogenomic Research Program and 2013 K000429 funded by the Korean Ministry of Science, ICT, and Future Planning. Study sponsor has no roles in the study design in the collection, analysis, and interpretation of data.

\section{DISCLOSURE/CONFLICT OF INTEREST}

The authors declare no conflict of interest.

1. Peek Jr RM, Fiske C, Wilson KT. Role of innate immunity in Helicobacter pylori-induced gastric malignancy. Physiol Rev 2010;90: 831-858.

2. Konturek PC, Bielanski W, Konturek SJ et al. Helicobacter pylori associated gastric pathology. J Physiol Pharmacol 1999;50:695-710.

3. Fox JG, Wang TC. Inflammation, atrophy, and gastric cancer. J Clin Invest 2007;117:60-69.

4. Wroblewski LE, Peek Jr RM, Wilson KT. Helicobacter pylori and gastric cancer: factors that modulate disease risk. Clin Microbiol Rev 2010;23: 713-739.

5. Blaser MJ, Berg DE. Helicobacter pylori genetic diversity and risk of human disease. J Clin Invest 2001;107:767-773.

6. Milne AN, Carneiro F, O'Morain C et al. Nature meets nurture: molecular genetics of gastric cancer. Hum Genet 2009;126:615-628.

7. Chan AO, Chu KM, Huang $\mathrm{C}$ et al. Association between Helicobacter pylori infection and interleukin 1beta polymorphism predispose to CpG island methylation in gastric cancer. Gut 2007;56:595-597.

8. Correa P. Human gastric carcinogenesis: a multistep and multifactorial process-First American Cancer Society Award Lecture on Cancer Epidemiology and Prevention. Cancer Res 1992;52:6735-6740.

9. Xia HH, Lam SK, Chan AO et al. Macrophage migration inhibitory factor stimulated by Helicobacter pylori increases proliferation of gastric epithelial cells. World J Gastroenterol 2005;11:1946-1950.

10. Peek Jr RM, Moss SF, Tham KT et al. Helicobacter pylori cagA+ strains and dissociation of gastric epithelial cell proliferation from apoptosis. J Natl Cancer Inst 1997;89:863-868.

11. Shigematsu $Y$, Niwa $T$, Rehnberg E et al. Interleukin-1beta induced by Helicobacter pylori infection enhances mouse gastric carcinogenesis. Cancer Lett 2013;340:141-147. 
12. Schumacher MA, Donnelly JM, Engevik AC et al. Gastric Sonic Hedgehog acts as a macrophage chemoattractant during the immune response to Helicobacter pylori. Gastroenterology 2012;142:1150-1159 e1156.

13. Shinohara ML, Lu L, Bu J et al. Osteopontin expression is essential for interferon-alpha production by plasmacytoid dendritic cells. Nat Immunol 2006;7:498-506.

14. Chang WL, Yang HB, Cheng $\mathrm{HC}$ et al. Increased gastric osteopontin expression by Helicobacter pylori Infection can correlate with more severe gastric inflammation and intestinal metaplasia. Helicobacter 2011;16:217-224.

15. Park JW, Park DM, Choi BK et al. Establishment and characterization of metastatic gastric cancer cell lines from murine gastric adenocarcinoma lacking Smad4, p53, and E-cadherin. Mol Carcinog; Available from: URL: onlinelibrary.wiley.com/doi/10.1002/mc.22226/abstract/ (in press).

16. Coligan JE. Current Protocols in Immunology. John Wiley and Sons: New York, p. v. (loose leaf).

17. Roussel $\mathrm{Y}$, Harris $\mathrm{A}$, Lee $\mathrm{MH}$ et al. Novel methods of quantitative realtime PCR data analysis in a murine Helicobacter pylori vaccine model. Vaccine 2007;25:2919-2929.

18. Lee IO, Kim JH, Choi YJ et al. Helicobacter pylori CagA phosphorylation status determines the gp130-activated SHP2/ERK and JAK/STAT signal transduction pathways in gastric epithelial cells. J Biol Chem 2010;285 $16042-16050$

19. Rogers AB, Taylor NS, Whary MT et al. Helicobacter pylori but not high salt induces gastric intraepithelial neoplasia in B6129 mice. Cancer Res 2005;65:10709-10715.

20. Kwon HJ, Won YS, Nam KT et al. Vitamin D(3) upregulated protein 1 deficiency promotes N-methyl-N-nitrosourea and Helicobacter pyloriinduced gastric carcinogenesis in mice. Gut 2012;61:53-63.

21. deFreitas D, Urbano M, Goulao $\mathrm{MH}$ et al. The effect of Helicobacter pylori infection on apoptosis and cell proliferation in gastric epithelium. Hepatogastroenterology 2004;51:876-882.

22. Fan XG, Kelleher D, Fan XJ et al. Helicobacter pylori increases proliferation of gastric epithelial cells. Gut 1996;38:19-22.

23. Luo JC, Shin VY, Yang YH et al. Tumor necrosis factor-alpha stimulates gastric epithelial cell proliferation. Am J Physiol Gastrointest Liver Physiol 2005;288:G32-G38.

24. Houghton J, Macera-Bloch LS, Harrison L et al. Tumor necrosis factor alpha and interleukin 1beta up-regulate gastric mucosal Fas antigen expression in Helicobacter pylori infection. Infect Immun 2000;68: 1189-1195.
25. Yamamoto T, Kita M, Ohno T et al. Role of tumor necrosis factor-alpha and interferon-gamma in Helicobacter pylori infection. Microbiol Immunol 2004:48:647-654.

26. Rahn W, Redline RW, Blanchard TG. Molecular analysis of Helicobacter pylori-associated gastric inflammation in naive versus previously immunized mice. Vaccine 2004;23:807-818.

27. Santos JC, Ladeira MS, Pedrazzoli Jr J et al. Relationship of IL-1 and TNF-alpha polymorphisms with Helicobacter pylori in gastric diseases in a Brazilian population. Braz J Med Biol Res 2012;45: 811-817.

28. Syu LJ, El-Zaatari M, Eaton KA et al. Transgenic expression of interferon-gamma in mouse stomach leads to inflammation, metaplasia, and dysplasia. Am J Pathol 2012;181:2114-2125.

29. Lund SA, Giachelli CM, Scatena M. The role of osteopontin in inflammatory processes. J Cell Commun Signal 2009;3:311-322.

30. Uede T. Osteopontin, intrinsic tissue regulator of intractable inflammatory diseases. Pathol Int 2011;61:265-280.

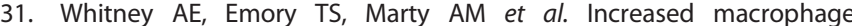
infiltration of gastric mucosa in Helicobacter pylori-infected children. Dig Dis Sci 2000;45:1337-1342.

32. Brenes F, Ruiz B, Correa P et al. Helicobacter pylori causes hyperproliferation of the gastric epithelium: pre- and post-eradication indices of proliferating cell nuclear antigen. Am J Gastroentero 1993;88:1870-1875.

33. Jones NL, Shannon PT, Cutz E et al. Increase in proliferation and apoptosis of gastric epithelial cells early in the natural history of Helicobacter pylori infection. Am J Pathol 1997;151:1695-1703.

34. Rokkas T, Ladas S, Liatsos C et al. Relationship of Helicobacter pylori CagA status to gastric cell proliferation and apoptosis. Dig Dis Sci 1999;44:487-493.

35. Beales IL. Effect of interlukin-1beta on proliferation of gastric epithelia cells in culture. BMC Gastroenterol 2002;2:7.

36. Meloche S, Pouyssegur J. The ERK1/2 mitogen-activated protein kinase pathway as a master regulator of the G1- to S-phase transition. Oncogene 2007;26:3227-3239.

37. Rittling SR, Matsumoto HN, McKee MD et al. Mice lacking osteopontin show normal development and bone structure but display altered osteoclast formation in vitro. J Bone Miner Res 1998;13. 1101-1111.

38. Rittling SR. Osteopontin in macrophage function. Expert Rev Mol Med 2011;13:e15. 